\title{
COMENTARIO EDITORIAL \\ Atención primaria y enfermería: La necesidad de una nueva especialidad
}

Recientemente fue publicado en el JAMA un artículo que describe el primer ensayo clínico randomizado y controlado que evalúa la práctica independiente de enfermeras entrenadas en el campo de la atención primaria en los EE.UU1 ${ }^{1}$. Este interesante estudio, realizado en 5 centros de salud dependientes del hospital académico de la Columbia University en Nueva York, asignó a un grupo de pacientes $(n=1316)$ que no contaban con un centro regular de atención a ser atendidos y seguidos por médicos o enfermeras de atención primaria. Los autores evaluaron la satisfacción, el estado de salud medido a través del SF-36 y pruebas fisiológicas de control de enfermedades crónicas como hipertensión arterial, diabetes y asma a los 6 meses y la utilización de servicios como visitas a los especialistas, consultas de atención primaria y de guardia e internaciones hasta un año después de la primera visita. Los grupos no mostraron diferencias en ninguno de los indicadores, mejoraron su estado funcional y el reporte de su estado de salud, aumentaron sus consultas programadas y disminuyeron las consultas de emergencia, además de presentar un alto grado de satisfacción. Los autores concluyen que, más allá de algunos detalles en el estudio que pueden limitar la generalización de sus hallazgos, este sustenta la hipótesis de que la calidad de la atención brindada por enfermeras o médicos no fue diferente usando el modelo tradicional de atención primaria. El estudio presenta algunas características que sin dudas limitan su validez externa, ya no solo con respecto a otros países sino dentro mismo de los EE.UU. La población fue seleccionada en salaśs de emergencias, entre pacientes de bajos recursos económicos que eran elegibles para recibir cobertura de salud a través del sistema de seguridad social (Medicaid). Eran en su mayoría mujeres inmigrantes hispanoparlantes casi exclusivamente de origen dominicano $(90 \%)$, a quienes se les ofrecía un sistema donde iban a acceder a los cuidados primarios de salud y utilizar el servicio sin costo alguno.

Si bien no hubo diferencias en las medidas fisiológicas elegidas, el tiempo de seguimiento de 6 meses es escaso para evaluar resultados en atención primaria.

Llamativamente, no se evaluaron actividades claramente identificadas con la atención primaria como las prácticas preventivas, n tampoco variables asociadas al proceso de atención tales como longitudinalidad, continuidad y adherencia al tratamiento, accesibilidad a la consulta y capacidad de resolución de situaciones en pacientes con múltiples problemas de salud.

Sox plantea que, si las conclusiones de este estudio fueran adoptadas como válidas, esto podría generar una situación de abierto conflicto con los médicos de atención primaria en el mercado laboral, ya que, dada la actual presión para contener el constante aumento de los gastos en salud, las instituciones podrían optar por incorporar más personal no médico entrenado en atención primaria con la idea de obtener resultados de igual calidad a menor costo ${ }^{2}$

En los EE.UU., desde hace más de 30 años, personal no médico entrenado para tal fin trabaja en las áreas ambulatorias de centros de salud municipales, universitarios y privados. Históricamente, la práctica era colaborativa y requería la supervisión directa de un médico que debía estar presente en el lugar para ser consultado y co-firmar las historias clínicas. Recientemente 22 estados han modificado la ley, permitiendo a los "nurse practitioners" realizar su actividad asistencial en forma independiente, y en aquellos que todavía requieren supervisión, ya no es necesario que el médico esté presente. Desde el gobierno federal, el Balanced Budget Act de 1997 autorizó a Medicare el pago directo por servicios a los nurse practitioners en centros no hospitalarios sin requerir supervisión médica.

Creo que las limitaciones metodológicas del estudio impiden sustentar las conclusiones de los autores en cuanto a su generaliza- ción, pero sin dudas abre la puerta a un debate más que interesante sobre el rol de la enfermería en la atención primaria de la salud. En EE.UU, una vez obtenido el título de grado (registered nurse) el entrenamiento de posgrado de los nurse practitioner los capacita para realizar todos los cuidados primarios, tanto de promoción como de prevención en todos los niveles, ser responsable del seguimiento del paciente, consultar directamente con los diferentes especialistas y, según la legislación de cada estado, prescribir drogas y modificar tratamientos.

Muchos países europeos han reformado sus sistemas de salud en los últimos años, intentando ampliar la cobertura y haciéndolos más accesibles, en especial a los grupos socialmente más vulnerables ${ }^{3}$. En todos ellos los profesionales médicos y no médicos de atención primaria se constituyeron en el eje del sistema de atención. En Latinoamérica, aún teniendo un gran camino por recorrer, algunos países como Chile han puesto énfasis en tratar de garantizar el acceso de la población a la atención primaria a través de centros de salud en los que la mayoría del personal no es médico.

Atención Primaria de la Salud (APS) implica el cuidado de la salud no solo individual, sino familiar y comunitaria a través de un sistema que sea accesible y equitativo y que represente un costo aceptable para la sociedad ${ }^{3}$.

También implica el trabajo coordinado en equipos de salud con otros profesionales y la relación con áreas de decisiones económicas y políticas que claramente exceden los límites específicos del accionar cotidiano de ese equipo de salud.

Es necesario incorporar una perspectiva amplia que considere a la comunidad. Por un lado los médicos no estamos formados para realizar diagnósticos comunitarios apropiados y muchas veces ignoramos factores socioeconómicos y culturales que son determinantes de algunos problemas de salud que vemos a diario. Por otro, el recurso médico esta muchas veces absorbido en la resolución de problemas de salud complejos, limitando el tiempo dedicado a tareas de educación, promoción y prevención, control y seguimiento (ambulatorio y domiciliario), etc. En un tercer escenario, mucho más dramático, el recurso médico simplemente no existe.

El uso apropiado, coordinado y eficiente del recurso humano debe ser una prioridad para alcanzar los objetivos definidos previamente. En nuestro país existen buenos ejemplos aislados de trabajo multidisciplinario, pero en general son realizados a costa de un gran esfuerzo individual de los participantes, ausencia de coordinación central y pobre reconocimiento tanto desde lo profesional como desde lo económico.

La Enfermería profesional debería jugar un rol protagónico en el equipo de salud complementando la función del médico en las tareas antes expuestas y permitiendo, de esta manera, expandir la capacidad asistencial y mejorar el acceso al sistema de salud.

Coordinar e integrar las tareas de los Enfermeros profesionales y los médicos en atención primaria es un desafío y es necesario generar espacios de formación de posgrado en el área cuyos objetivos puedan enmarcarse en las leyes vigentes del ejercicio de la profesión.

La ley 24.004 del ejercicio de la Enfermería, promulgada en 1991 y reglamentada en 1993 define textualmente que "el ejercicio de la Enfermería comprende las funciones de promoción, recuperación y rehabilitación de la salud, asi como la de prevención de enfermedades, realizadas en forma autónoma dentro de los límites de competencia que deriva de las incumbencias de los respectivos títulos habilitantes". La reglamentación del artículo 3ro intenta definir más 
específicamente las funciones y competencias del nivel profesional.

El artículo $7^{\circ}$ de la ley 24.004 define las condiciones que se pueden acreditar para que los enfermeros puedan utilizar el título de especialistas. Si bien estas son muy diversas y no todas implican requisitos equivalentes en cuanto al grado de formación, una de las alternativas claramente estipuladas es una residencia de al menos 3 años.

En Argentina, hasta hoy, no hay especialidades oficialmente reconocidas en enfermería.

Recientemente la legislatura de la Ciudad de Buenos Aires votó por unanimidad la ley 298 de Ejercicio de la Enfermería, que establece en su artículo 3 ro el cuidado de la salud en todo el ciclo vital de la persona, familia, comunidad y su entorno a través de intervenciones libres, autónomas, independientes e interdependientes.

En principio las leyes pueden interpretarse ampliamente y no parecería haber expresas dificultades para proponer e implementar programas de formación.
Idealmente, la especialidad de Enfermería en Atención primaria debería estar limitada a los profesionales universitarios (título de grado) que acrediten una residencia de 3 años. El programa de una residencia en atención primaria debería poner especial énfasis en los conocimientos teórico prácticos que permitan al enfermero/a adecuadamente entrenado desarrollar una práctica supervisada en el ámbito individual y comunitario, adquirir responsabilidades progresivas y asumir, en forma coordinada con los médicos, la atención de poblaciones definidas.

El Estado Nacional, Las provincias y los municipios deberían garantizar los recursos financieros para llevar adelante estos programas. Creemos que en el contexto actual de necesidad de reforma del sistema de salud, contemplar esta propuesta en un país en el que un $40 \%$ de la población no accede a una cobertura en salud y el recurso médico dedicado a la atención primaria no llega al 30\%; la formación de un recurso idóneo que pueda integrar y complementar sus funciones ayudará a dar mayor respuesta a los sectores más vulnerables y logrará un sistema más accesible y equitativo.

Dra. Mariela Alderete

Centro de Medicina Asistencial y Solidaria.

Dr. Fernando Rubinstein

Unidad de medicina familiar y Preventiva. Hospital Italiano de Buenos Aires

\section{Referencias}

1- Mundinger M, Kane R. Primary Care outcomes in patients treated by Nurse Practitioners or Physicians. JAMA, 2000:283;59-68.

2- H.Sox. Independent Primary care Practice by Nurse Practitioners JAMA, 2000:283;106-108

3- Rubinstein A. Reforma de la Salud centrada en la Atención Primaria: Un cambio impostergable para la atención médica Argentina. Comunicación Personal, datos no publicados.

4- Ley 24.004 de Ejercicio de la Enfermería de la República Argentina

5- Ley 298 de Ejercicio de la Enfermería de la Ciudad de Buenos Aires. 